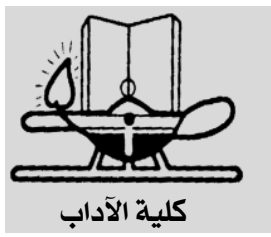

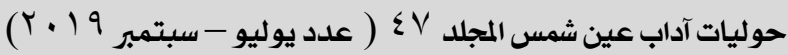
http://www.aafu.journals.ekb.eg

(دورية علمية محكمة)

\title{
تشاكل الألفاظبالإمابقة
}

*الاد بن عليّ بن عبد الله الصبحيّ

قسم اللغة العربية

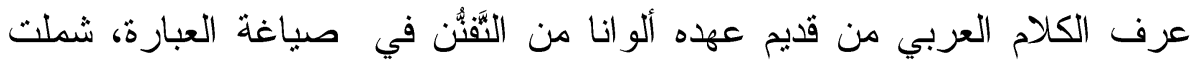

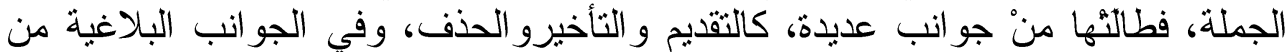

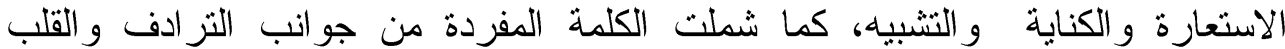

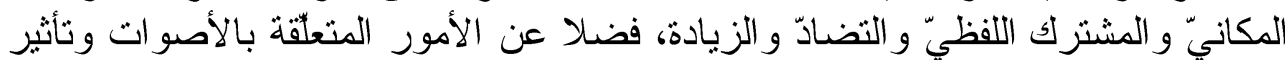

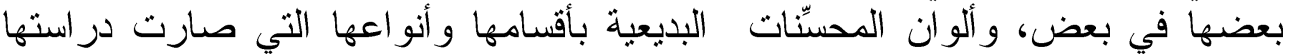
جزءا أساسيا في الدرس البلاغيّ.

و أدلى اللغويون بدلو هم في البحث الذيّي يتئاول، بالإضافة إلى أمور القاعدة النحوية

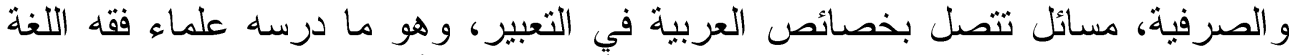

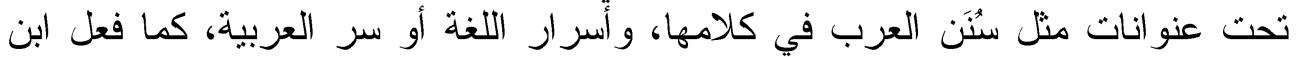

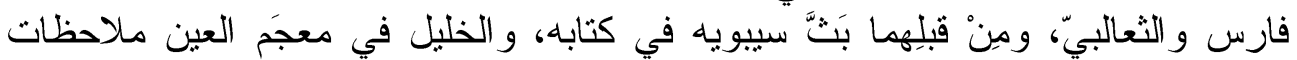

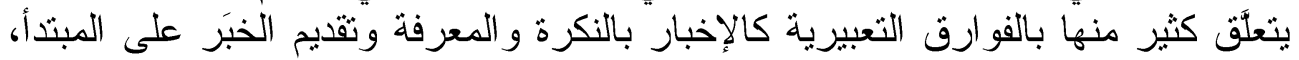

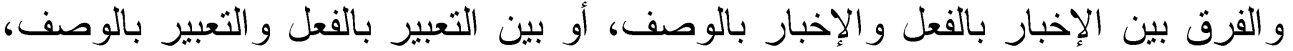

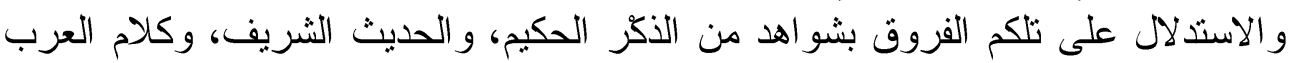
شعره و منثور ه.

وقد جرى التطبيق في البحث على نماذج من الكلام العربيّ الفصيح في مستوياته

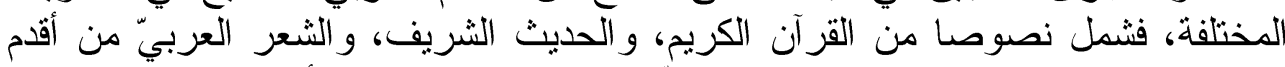

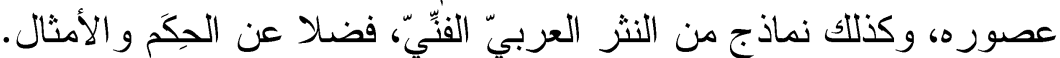

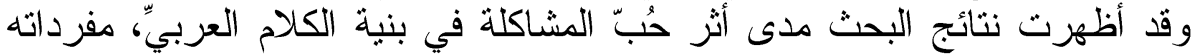

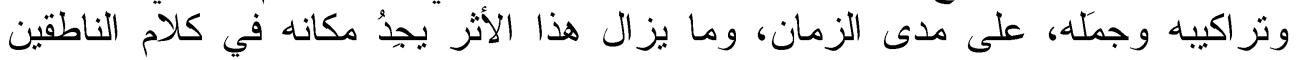

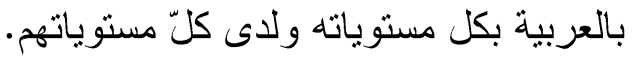




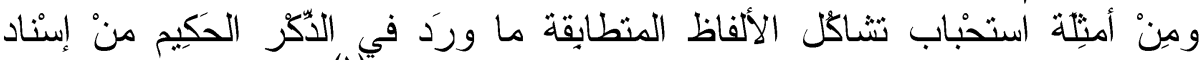

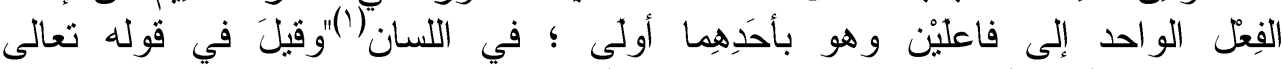

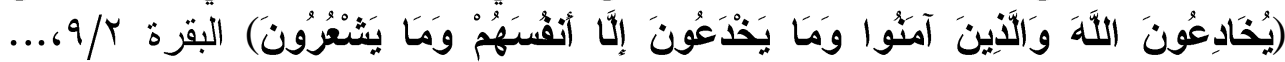

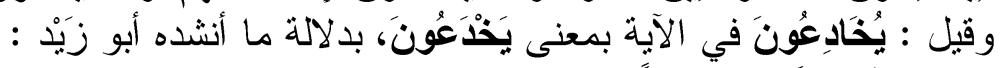

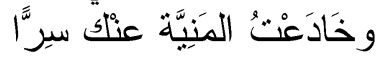

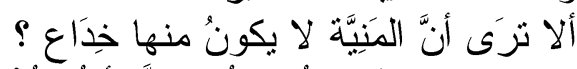

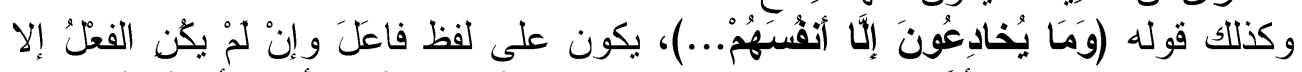

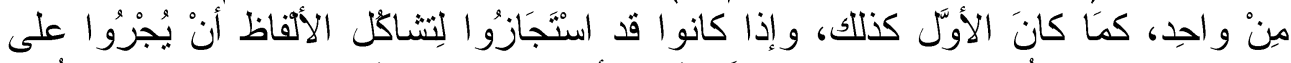

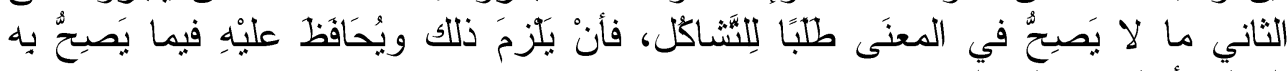

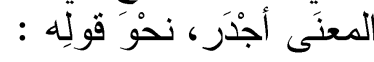

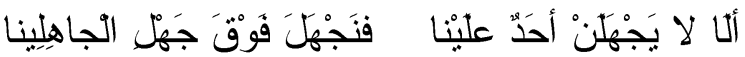

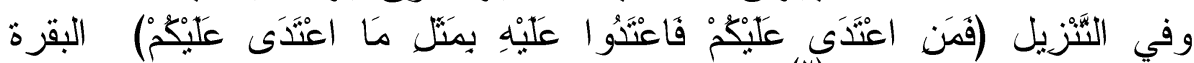

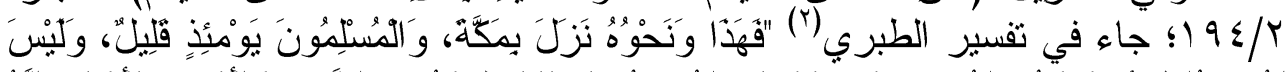

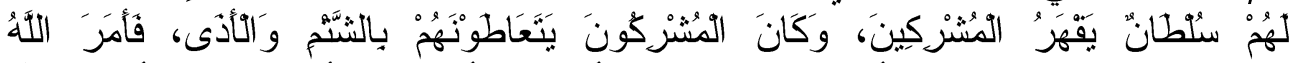

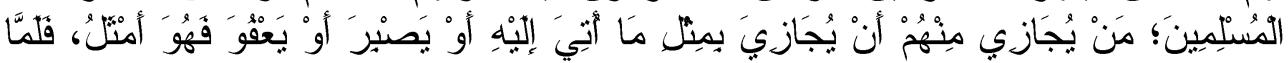

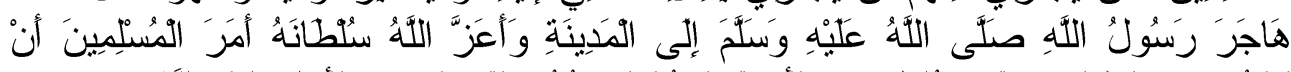

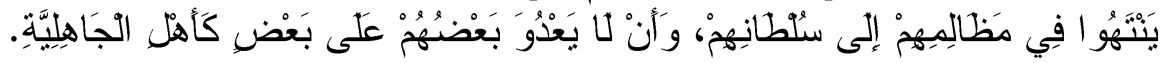

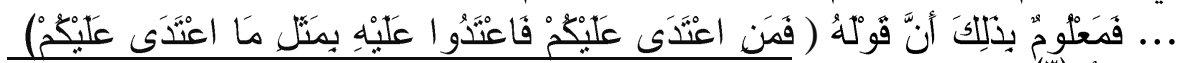

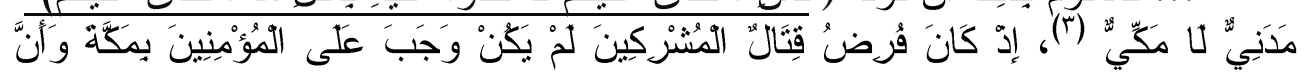

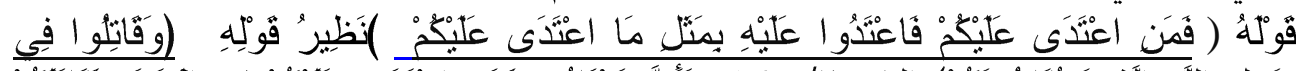

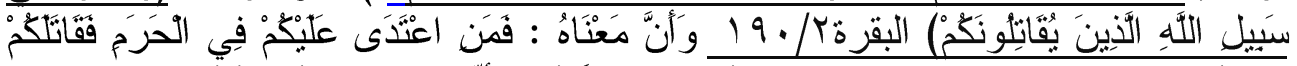

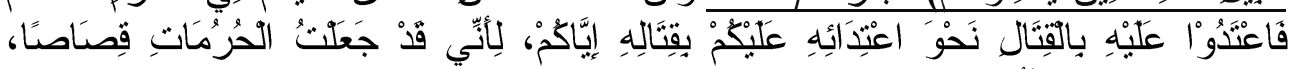

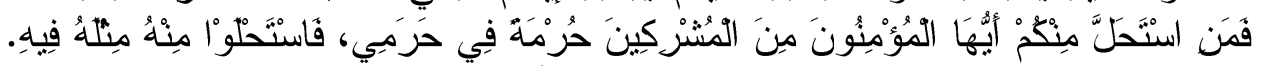

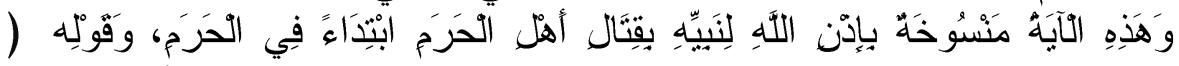

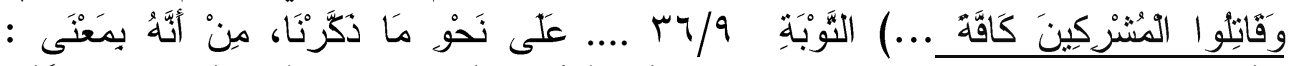

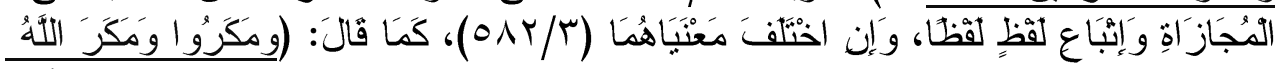

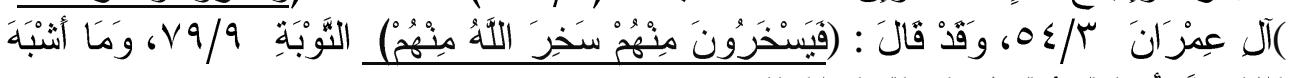

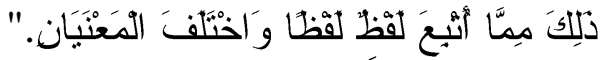
في الشعر والرَّجز

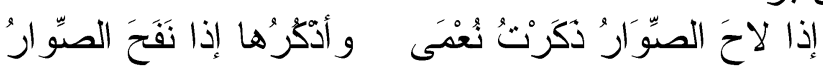

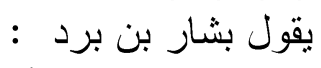

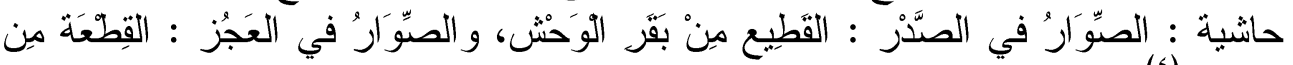

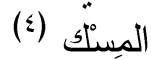

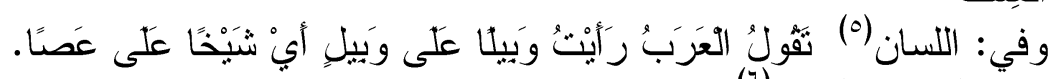

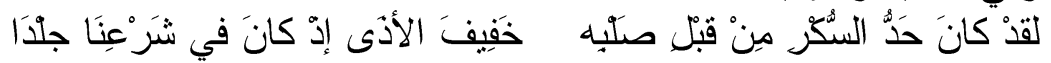

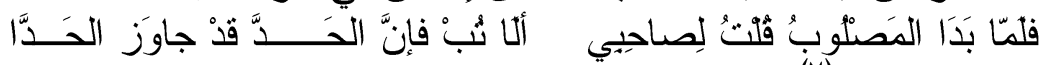

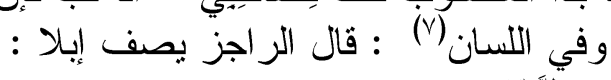

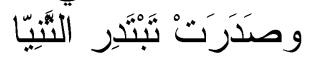
تَرَبَ مِنْ دُعْيِِِها ذُعْمِيّا 
دُعْيَّها : وَسَطها، دُعْيَّا : أب طريقَا مَوُْطو عا.

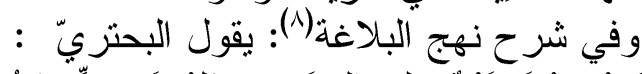

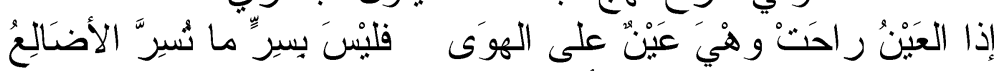

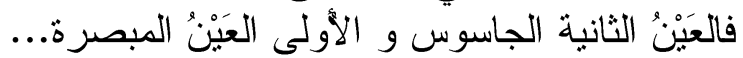

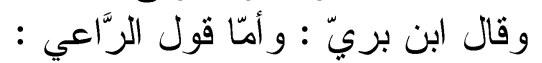

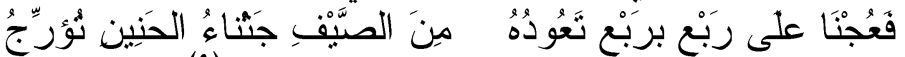

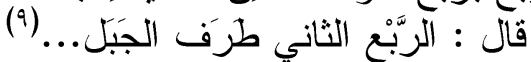

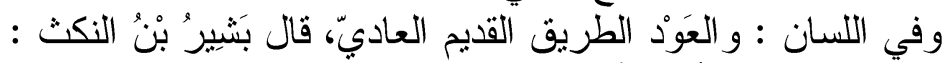

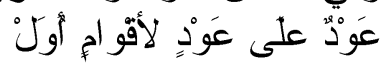

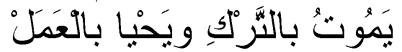

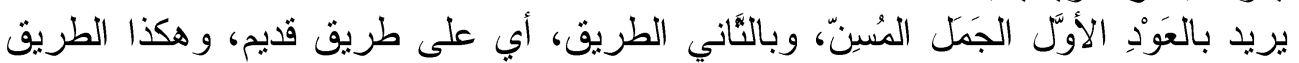

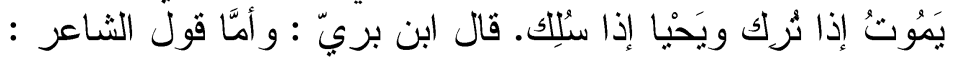

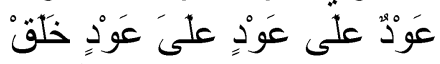

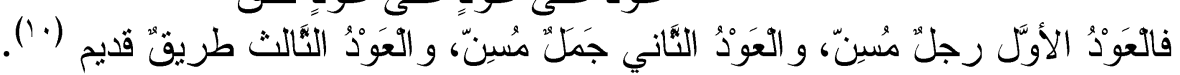

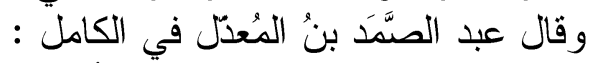

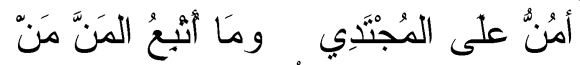

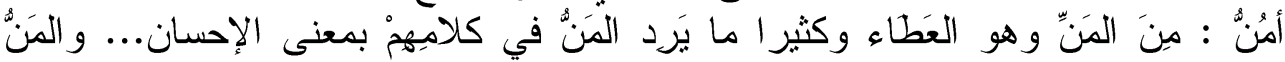

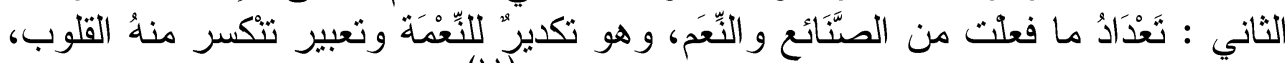

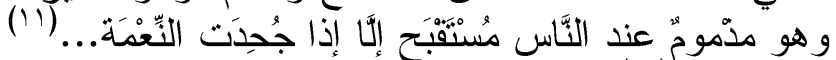

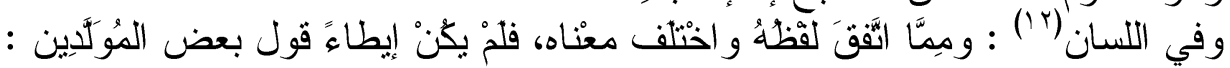

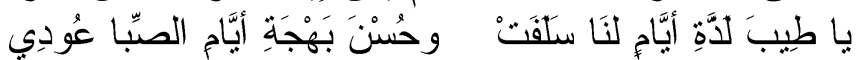

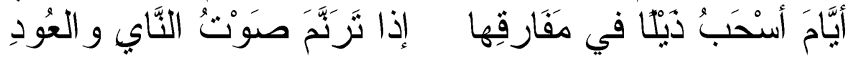

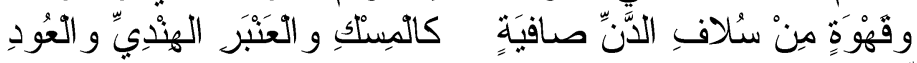

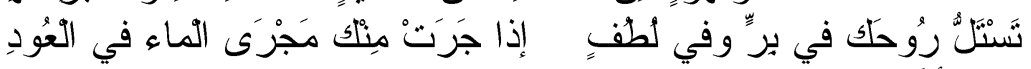

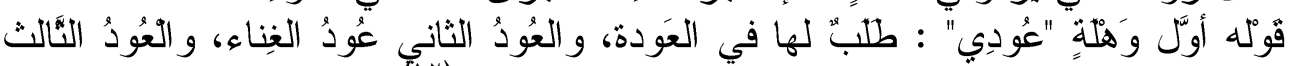

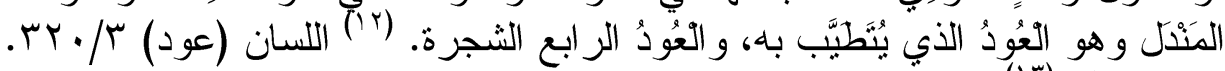

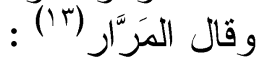

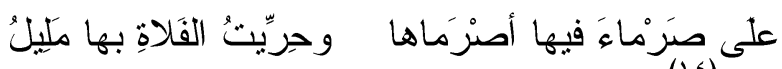
ويقول النابغة الذبياني (؟) : :

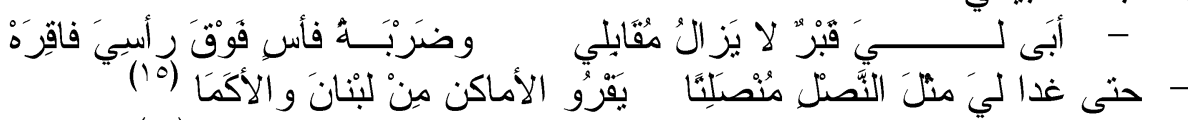

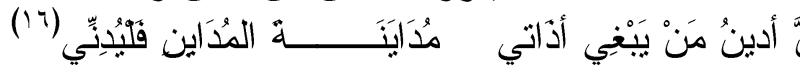

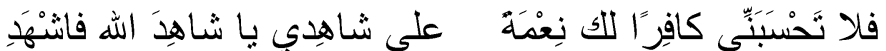

ويقول الأعشَى (iv)

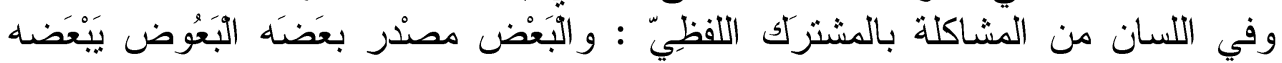

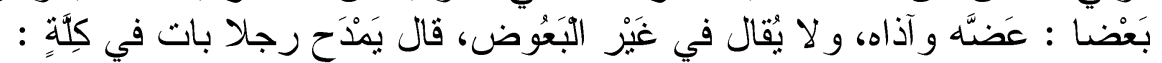

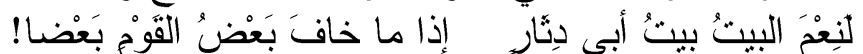

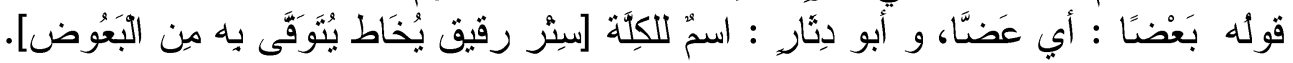

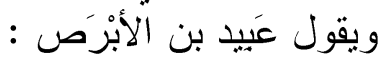

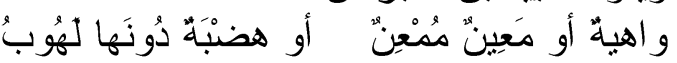

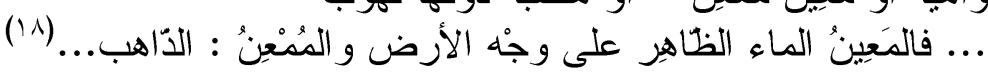




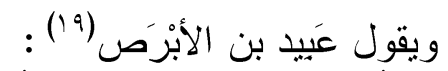

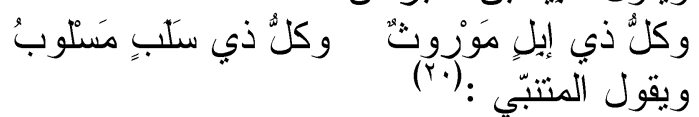

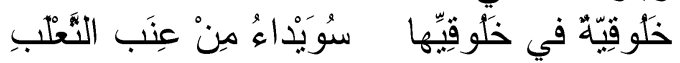

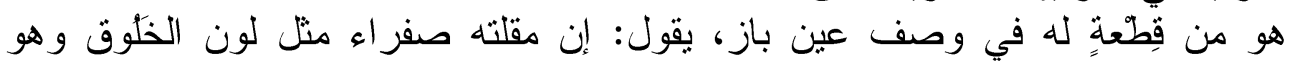

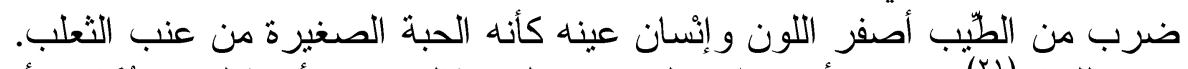

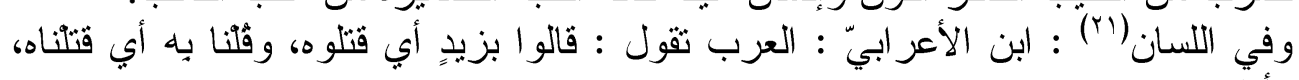
و وأنشد:

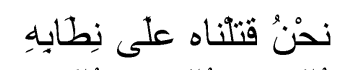

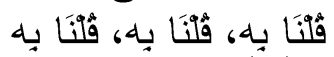

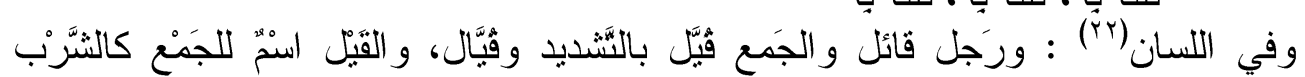

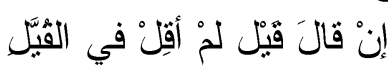

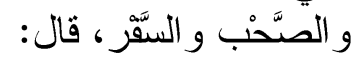

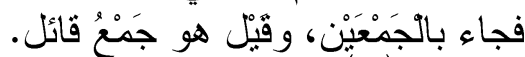

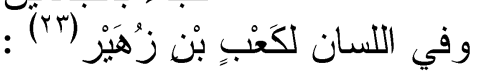

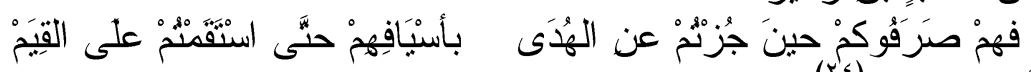

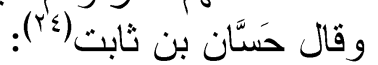

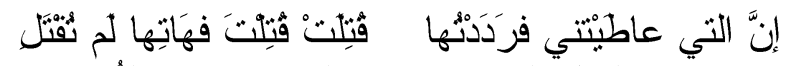

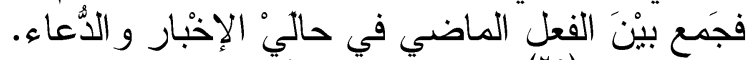

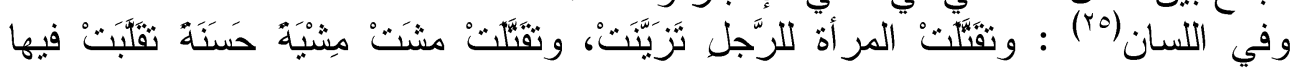

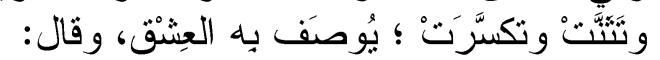

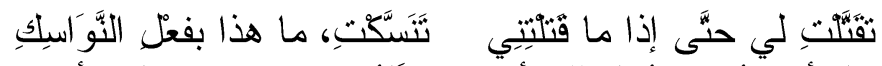

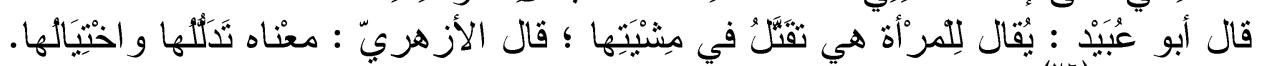
وفي اللسان(بَ):

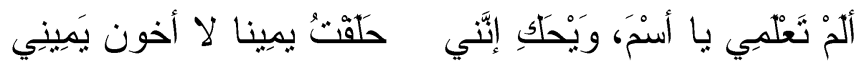

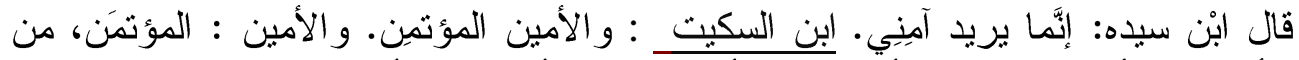

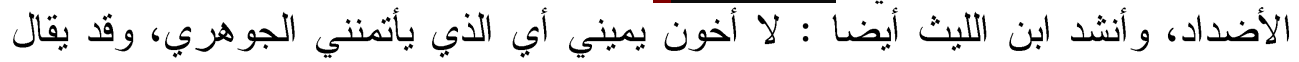

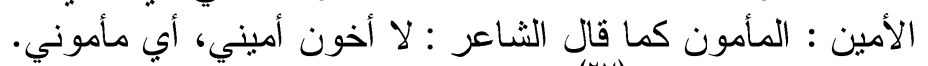

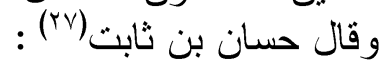

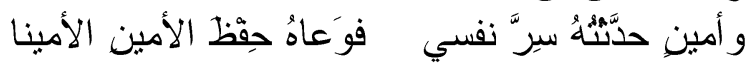

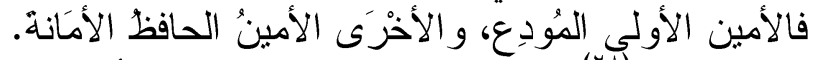

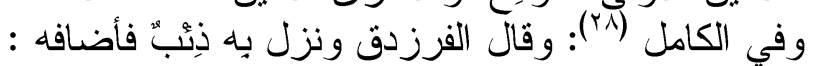

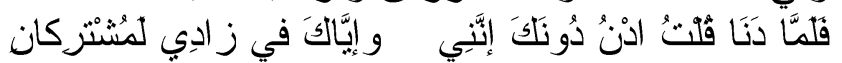

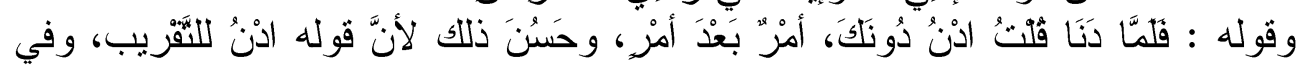

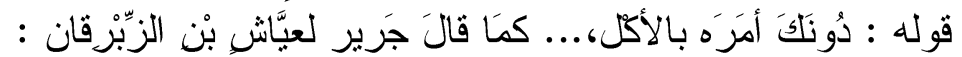

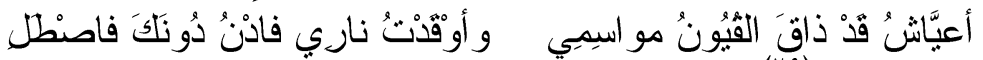

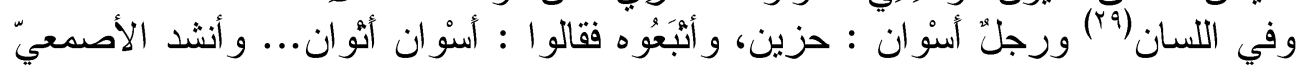

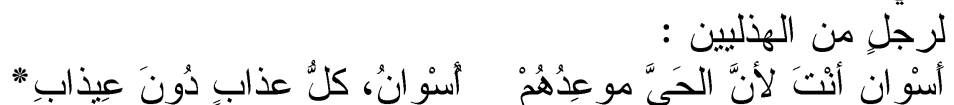

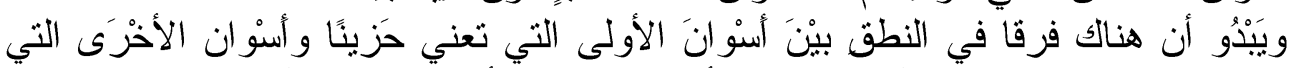

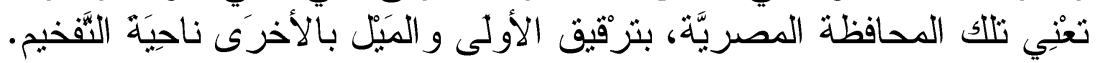




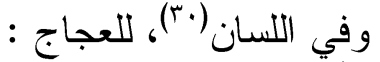

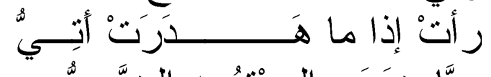

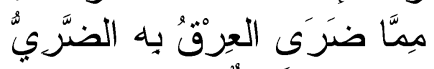

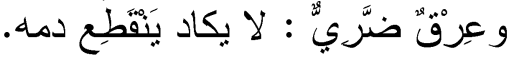

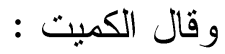

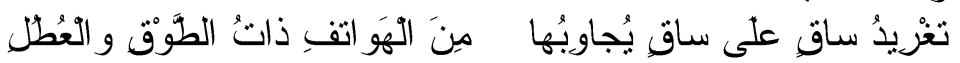

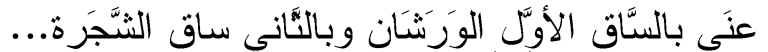

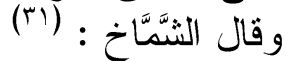

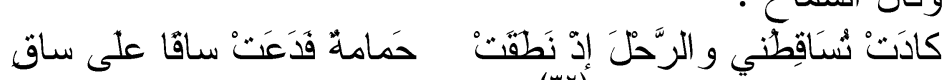

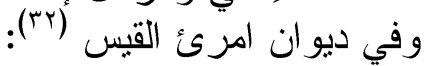

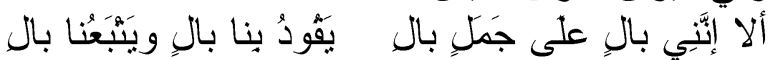

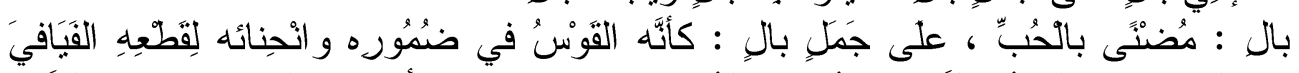

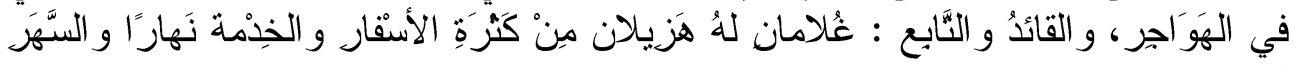

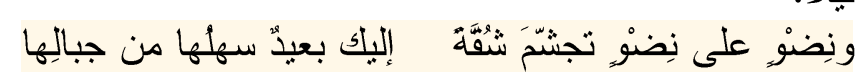
وقوله " ونضو على نضو تجشم شقة " فهو معنى مليح جيد، وهو كثير في أشعار هم، فمن ذلك قول العباس بن الأحنف:

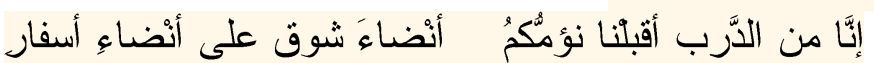

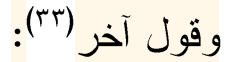

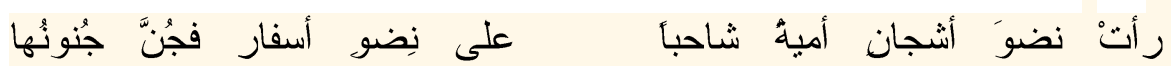

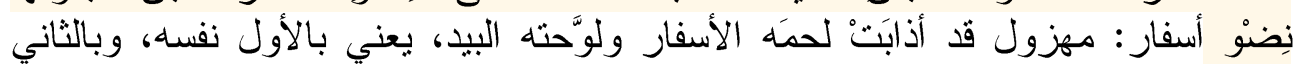

\section{وقال آخر: (ك)}

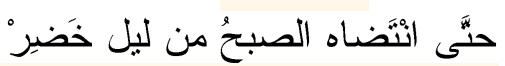

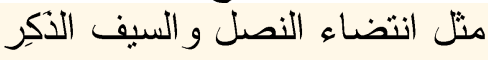

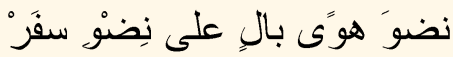

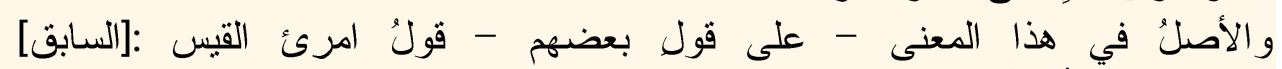

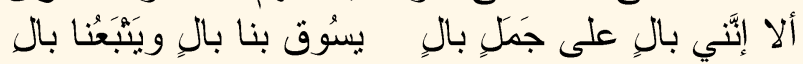

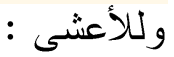

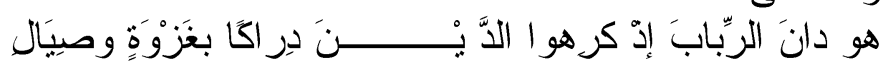

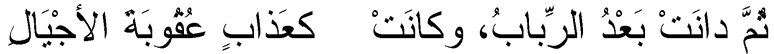

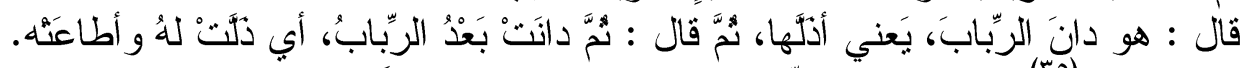

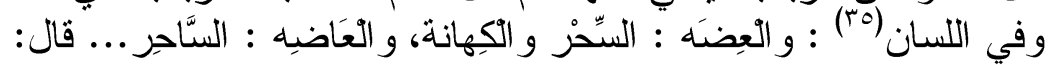

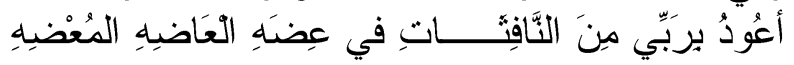

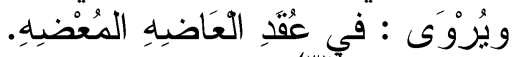

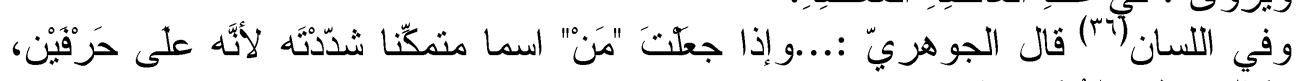

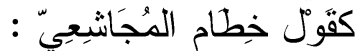

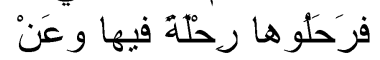

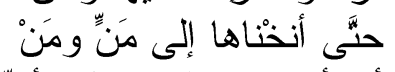

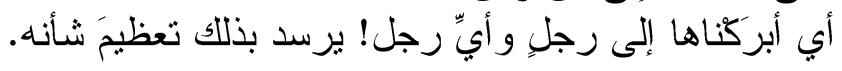




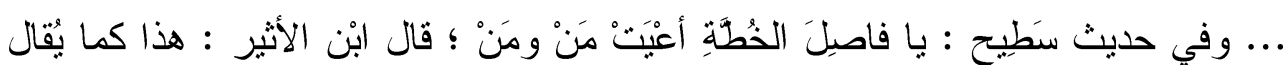

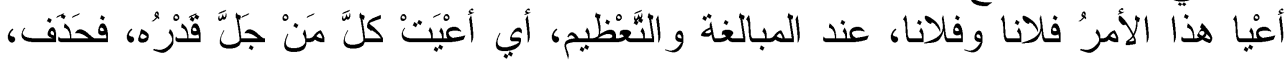

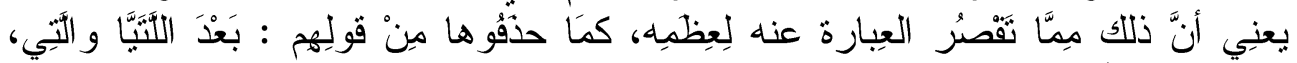

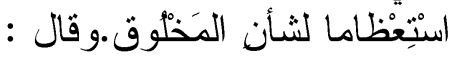

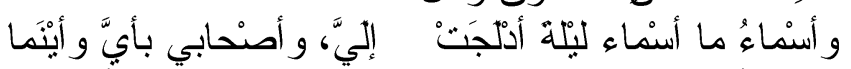

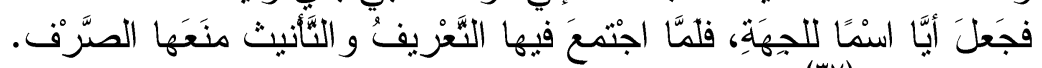

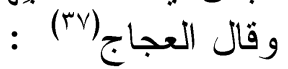

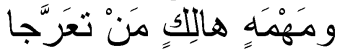

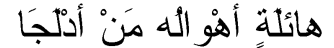

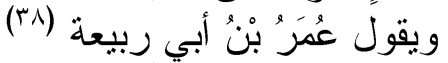

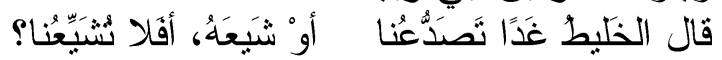

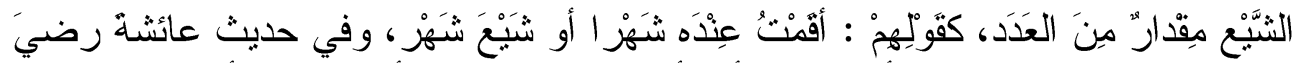

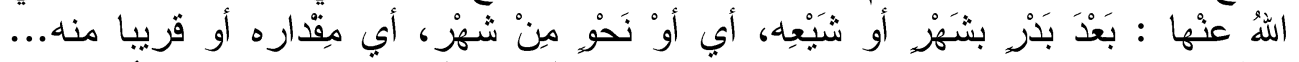

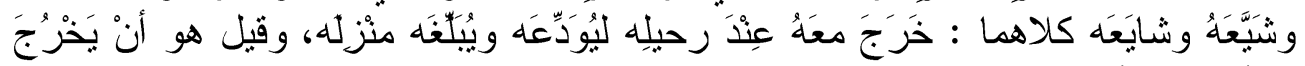

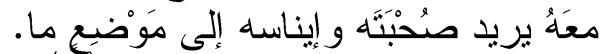

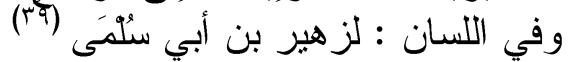

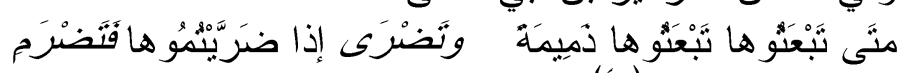

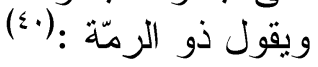

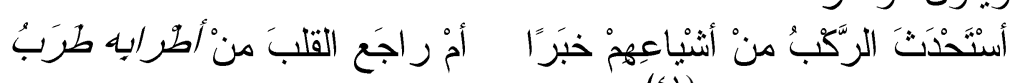

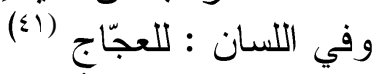

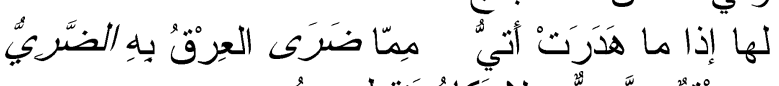

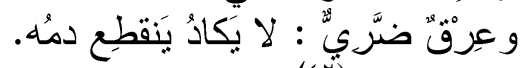

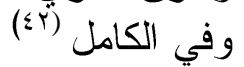

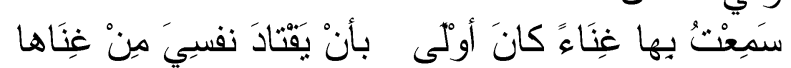

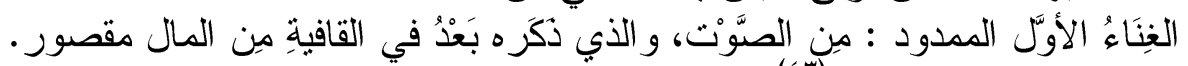

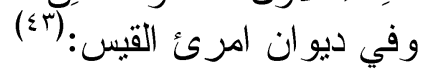

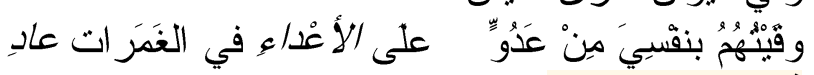

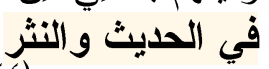

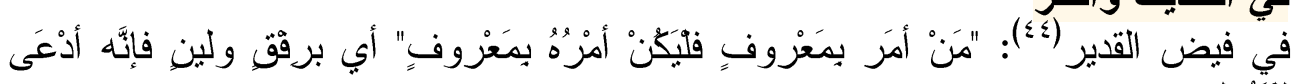

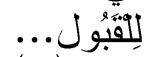

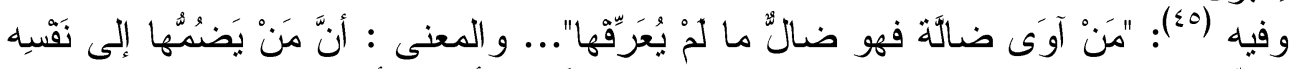

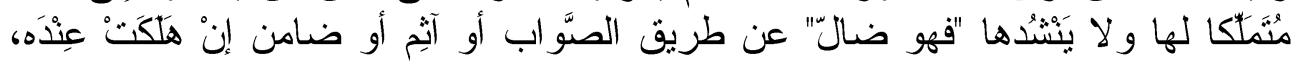

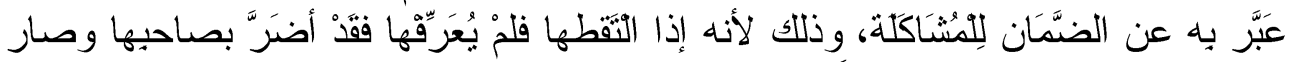

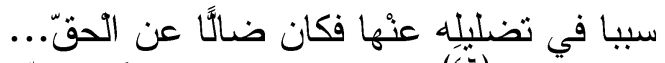

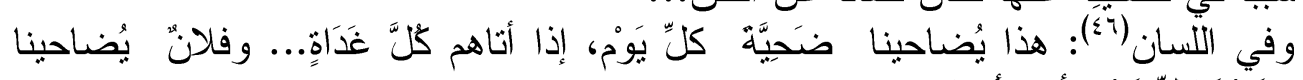

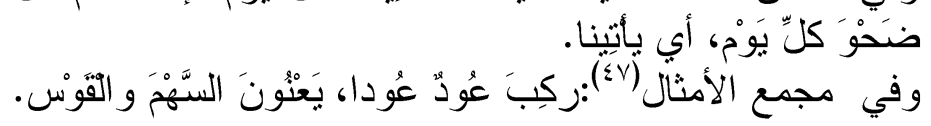




\section{Abstract \\ Formation of words by matching \\ By Khalid bin Ali bin Abdullah Al-Subhi}

Arabic speech was known from ancient times in terms of sophistication in the formulation of the phrase, including the sentence, and it took hold in many aspects, such as introduction, delay and deletion, and in the rhetorical aspects of metaphor, metaphor and simile, as well as the singular word included aspects of synonymy, spatial heart, common verbal, contradiction and addition, as well as related matters With the sounds and influence of each other, and the colors of the exquisite enhancers, with their sections and types, whose study became an essential part of the rhetorical lesson.

Linguists have made their substitution in the research that deals, in addition to grammatical and morphological matters, issues related to the characteristics of Arabic in expression, which scholars of jurisprudence studied under titles such as the Sunan of the Arabs in their words, and the secrets of the language or the secret of Arabic, as Ibn Faris and Al-Tha'alabi did, and before them Sibawayh, in his book, Hebron in Al-Ain's dictionary, transmitted notes, many of which relate to expressive differences, such as news of negligence, knowledge, and presentation of news on the beginner, and the difference between news already and news in description, or between expression already and expression in description, and inferring these differences with evidence from wise remembrance, hadeeth, and speech Arabs are his hair and strewn.

The research was applied to examples of eloquent Arabic speech at its various levels, including texts from the Noble Qur'an, hadith, and Arabic poetry from its earliest ages, as well as examples of Arab artistic prose, as well as wisdom and proverbs.

The results of the research showed the extent of the impact of the love of the problem on the structure of Arabic speech, its vocabulary, its structures and its beauty, over time, and this effect still finds its place in the speech of Arabic speakers at all levels and at all levels.

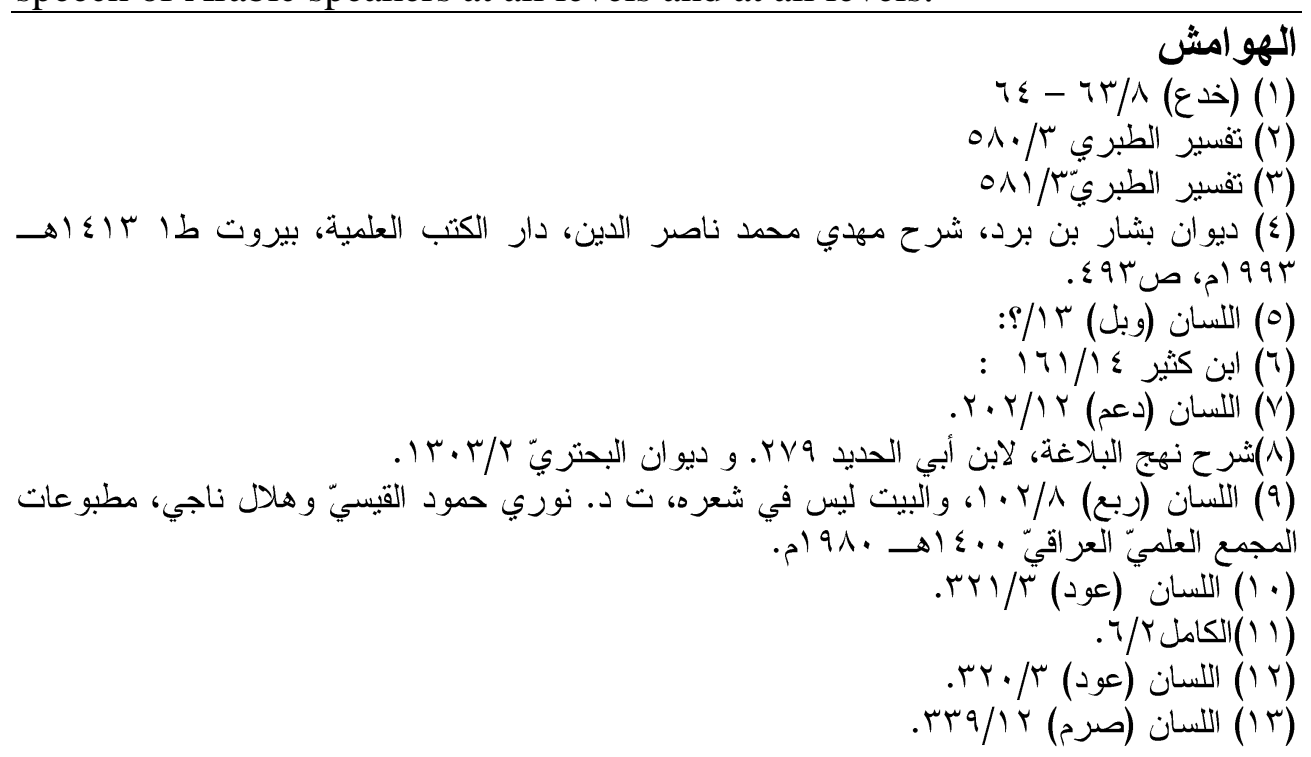




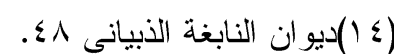

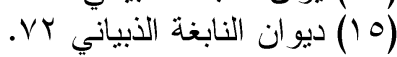

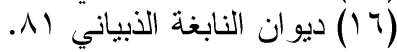

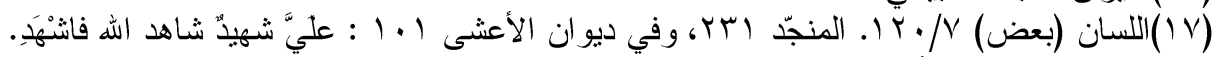

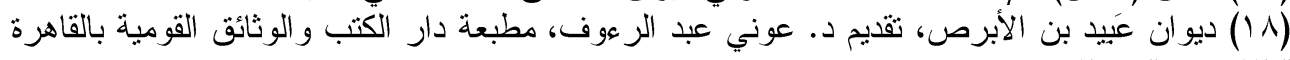

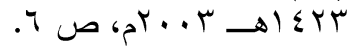

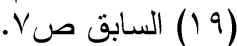

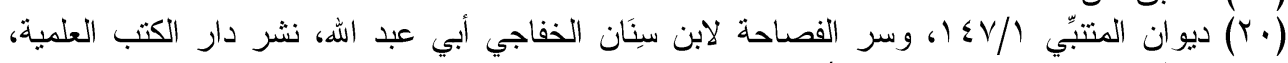

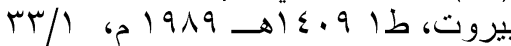

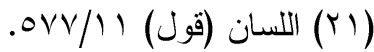

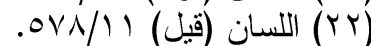

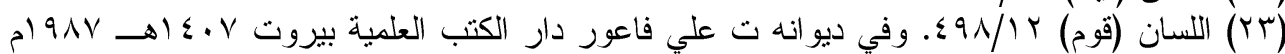

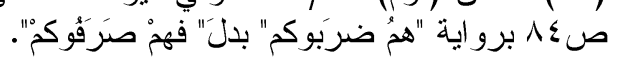

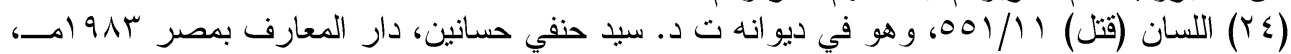

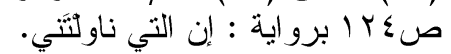

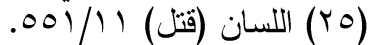

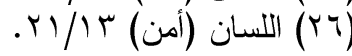
r

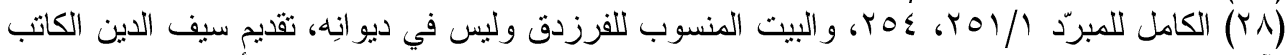

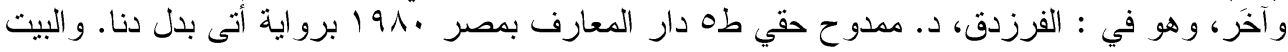

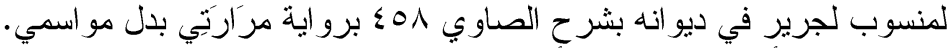

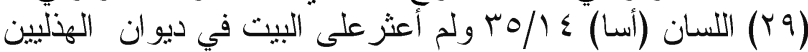

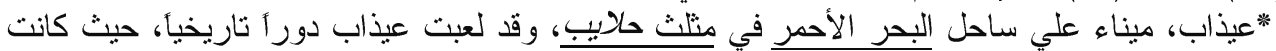

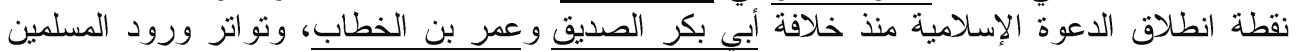

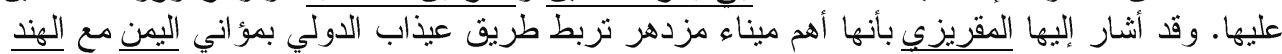

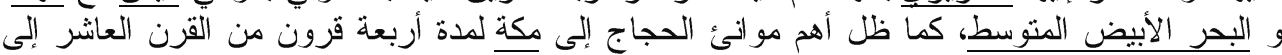

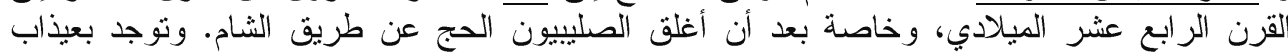

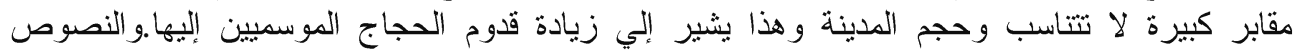

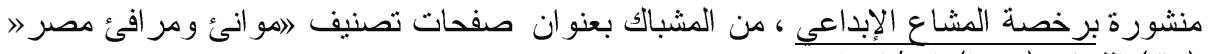

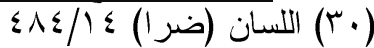

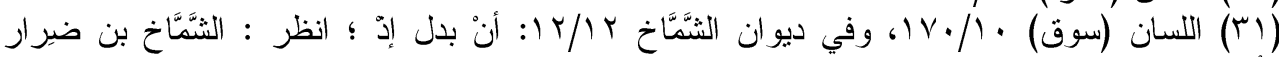

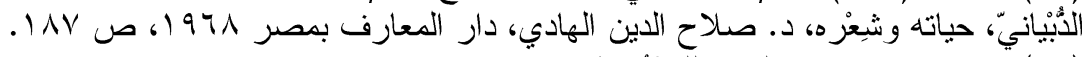

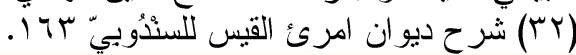

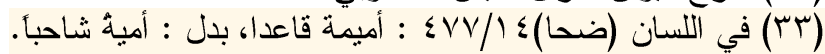

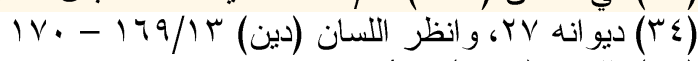
(

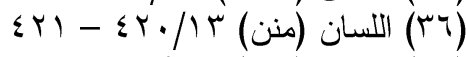

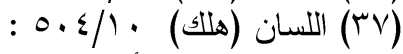

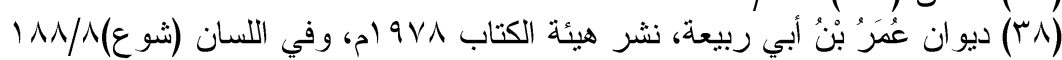

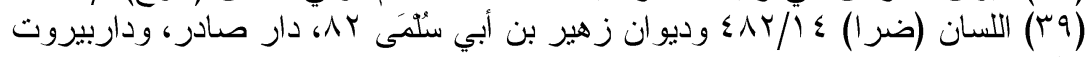

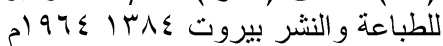

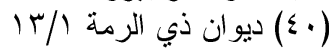

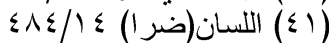

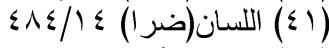

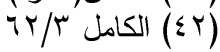
( )

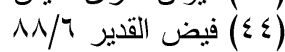

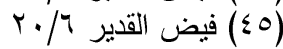

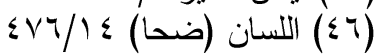

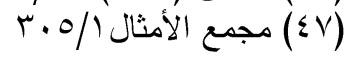

